\title{
Ion-pair Reversed-phase Liquid Chromatographic Separation of Oligonucleotides
}

\author{
Takumi Takano,* Chiaki Aoyama,* Yoshiro Terasaki,* Kenichi Suzuki,* Aki Ando,* \\ Yanting Song, ${ }^{* *}$ and Makoto TsunODA***† \\ *GL Sciences Inc., 6-22-1 Nishi Shinjuku, Shinjuku, Tokyo 163-1130, Japan \\ **School of Pharmaceutical Sciences, Hainan University, Haikou 570228, China \\ ***Graduate School of Pharmaceutical Sciences, University of Tokyo, 7-3-1 Hongo, Bunkyo, Tokyo 113-0033, \\ Japan
}

\begin{abstract}
Therapeutic oligonucleotides have recently been approved in the United States, the EU, and Japan. Hence, the analysis of oligonucleotides is an important topic in drug development. Liquid chromatographic techniques are commonly used for purity verification and the determination of oligonucleotides. In ion-pair reversed-phase separation, several parameters, such as the pore size of the stationary phase, mobile phase additives, and column temperature, were investigated using three types of oligonucleotides $(18,19$, and 20 mer). All of the investigated parameters could influence the separation, and they are expected to be useful for optimizing oligonucleotide separation.
\end{abstract}

Keywords Pore size, mobile phase additives, column temperature, DNA, HPLC

(Received July 22, 2021; Accepted August 24, 2021; Published December 10, 2021)

\section{Introduction}

Synthetic oligonucleotides have recently been used as potential therapeutic agents to control gene expression and suppress the formation of specific proteins..$^{1,2}$ There are several kinds of synthetic oligonucleotides, such as antisense oligonucleotides, small-interfering RNAs (siRNAs), and aptamers, which are used to treat cancer, viral infections, Alzheimer's disease, genetic disorders, etc. In 1998, the first antisense oligonucleotide, fomivirsen (21 mer oligonucleotides), was approved by the Food and Drug Administration (FDA) for treating cytomegalovirus retinitis. ${ }^{3}$ As of December 2020, there were 12 FDA-approved oligonucleotide drugs, ${ }^{4}$ some of which have also been approved in the EU and Japan. Besides, there are many oligonucleotidebased drugs in development and clinical trials.

Oligonucleotides are typically prepared by a stepwise reaction based on phosphoramidite chemistry with the aid of a fully automated solid-phase synthesizer. Owing to the large number of steps involved in this process and the high ( $\sim 99 \%)$ coupling efficiency in each step, impurities and degradation products are formed. It is imperative to remove these undesired compounds and characterize the desired product for therapeutic use. Hence, oligonucleotides are typically subjected to purification following the synthesis.

A number of chromatographic methods, including ionexchange chromatography, ${ }^{5}$ ion-pair reversed-phase liquid chromatography (LC), ${ }^{6,7}$ mixed-mode (reversed-phase and ionexchange) $\mathrm{LC}^{8}{ }^{8}$ and hydrophilic interaction chromatography (HILIC), ${ }^{9,10}$ have been reported for the analysis and purification

\footnotetext{
† To whom correspondence should be addressed.
}

E-mail: makotot@mol.f.u-tokyo.ac.jp of oligonucleotides. Among them, ion-pair reversed-phase LC is a commonly used technique for the separation of oligonucleotides because it is easily interfaced with mass spectrometry with volatile mobile phase additives. ${ }^{11}$ In this method, negatively charged oligonucleotides interact with positively charged alkylammonium ions to allow for strong retention of the former on a reversed-phase stationary phase. Many studies have shown that several factors are significant for separating the oligonucleotide ladder. ${ }^{7,12}$ Those for impurity assays, which should separate single-nucleotide differences, are not fully understood. Hence, this study examined several parameters, namely, the pore size of the stationary phases, hexafluoroisopropanol (HFIP) addition to mobile phases, the column temperature, and type of stationary phases, for oligonucleotide separation by ion-pair reversed-phase LC.

\section{Experimental}

Reagents and chemicals

Single-stranded DNA oligonucleotides, 5'-CATGACGTTCCTGATGCT-3' (18 mer), 5'-CCATGACGTTCCTGATGCT-3' (19 mer), and 5'-TCCATGACGTTCCTGATGCT-3' (20 mer), were purchased from Gene Design Inc. (Osaka, Japan) and then reconstituted in water. All of the samples were prepared at a concentration of $10 \mathrm{nM}$.

Hexafluoroisopropanol (HPLC grade) was obtained from FUJIFILM Wako Pure Chemical (Osaka, Japan). Acetonitrile (HPLC grade) was purchased from Kishida Chemical (Osaka, Japan). All other reagents were of analytical grade.

\footnotetext{
$L C$ conditions

A GL7700 HPLC system (GL Sciences, Tokyo, Japan) was
} 
(a)

(b)

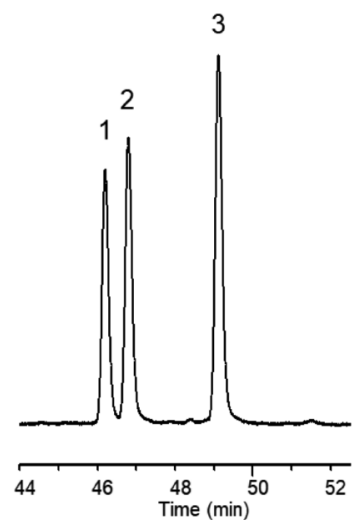

(c)

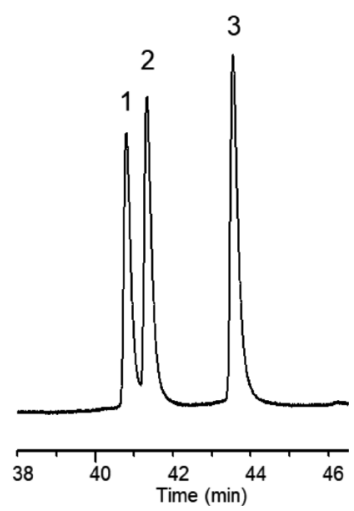

Fig. 1 Effects of pore size of the stationary phases on the separation of three oligonucleotides: (a) $100 \AA$ (IntertSustain C18), (b) $200 \AA$ (IntertSustainSwift C18), and (c) $300 \AA$ (Inertsil WP300 C18). Peaks: 1, 5'-CATGACGTTCCTGATGCT-3' (18 mer); 2, 5'-CCATGACGTTCCTGATGCT-3' (19 mer); 3, 5'-TCCATGACGTTCCTGATGCT-3' (20 mer).

used for LC. Separation was performed on four types of reversed-phase columns (GL Sciences), InertSustain C18 (3 $\mu \mathrm{m}$, $3.0 \times 100 \mathrm{~mm})$, InertSustainSwift C18 $(3 \mu \mathrm{m}, 3.0 \times 100 \mathrm{~mm})$, Inertsil WP300 C18 $(5 \mu \mathrm{m}, 3.0 \times 100 \mathrm{~mm})$, and InertSustainSwift C8 $(3 \mu \mathrm{m}, 3.0 \times 100 \mathrm{~mm})$. The flow rate of the mobile phase was $0.6 \mathrm{~mL} / \mathrm{min}$. A binary gradient elution program was then adopted. Mobile phase A consisted of $5 \mathrm{mM}$ triethylammonium acetate (TEAA; $\mathrm{pH} 6.5$ ), and mobile phase B consisted of $5 \mathrm{mM}$ TEAA $(\mathrm{pH} 6.5) /$ acetonitrile $(80: 20, \mathrm{v} / \mathrm{v})$. The linear gradient was $5-50 \% \mathrm{~B}$ over $60 \mathrm{~min}$. The column temperature was maintained at 40 or $60^{\circ} \mathrm{C}$. The injection volume was $1 \mu \mathrm{L}$. UV detection was performed at a wavelength of $260 \mathrm{~nm}$.

\section{Results and Discussion}

Most FDA-approved oligonucleotides are 18 - 30 mer; hence, 20 mer oligonucleotides (for chemical composition, see Materials and Methods section) were used as typical oligonucleotides. The 18 and 19 mer oligonucleotides were used as impurities. The effects of several parameters, such as the pore size of the stationary phase, mobile phase additives, column temperature, and type of stationary phase, were investigated for separating three types of oligonucleotides (18, 19, and 20 mer).

\section{Effects of pore size of the stationary phase}

First, the pore size of the reversed-phase column was investigated. In porous silica particles, most interactions of the analytes with stationary phases occur in the pores. For lowmolecular-weight compounds (molecular weight $<1000 \mathrm{Da}$ ), the average pore size used is $100 \AA$. Large-pore particles $(\sim 300 \AA)$ are used for separating large molecules, such as proteins. Considering that the molecular weights of oligonucleotides used in this study were about $6000 \mathrm{Da}$, which is greater than that of low-molecular-weight compounds, but smaller than that of proteins, the pore size should influence the retention and separation. In this study, three types of reversed-phase columns with pore sizes of $100 \AA$ (IntertSustain C18), $200 \AA$ (IntertSustainSwift C18), and $300 \AA$ (Inerstil WP300 C18) were investigated. The separation results are shown in Fig. 1. The separation efficiency was poor for a pore size of $100 \AA$
(Fig. 1(a)), probably because these pores were too small for the oligonucleotides investigated. Good separation was achieved with pore sizes of 200 and $300 \AA$, and a difference in retention was observed (Figs. 1(b) and 1(c)). The column with a pore size of $300 \AA$ led to weaker retention than that with a pore size of $200 \AA$. This difference can be explained by the surface area of the particles. Columns with pore sizes of 200 and $300 \AA$ had surface areas of 200 and $150 \mathrm{~m}^{2} / \mathrm{g}$, respectively. Accordingly, a column with a pore size of $200 \AA$, which offered better separation, especially for 18 and 19 mer, was used in subsequent experiments.

\section{Effects of hexafluoroisopropanol addition to mobile phases}

Ion-paring reagents are indispensable for the retention and separation of oligonucleotides in reversed-phase columns. However, in the case of detection, increasing the concentration of the ion-pairing reagent does not degrade the separation resolution, but decreases the sensitivity in mass spectrometry (MS) detection. Apffel et al. reported that the addition of hexafluoroisopropanol (HFIP) to the mobile phase can enhance the sensitivity of MS with electrospray ionization. ${ }^{13}$ Herein, we investigated the manner in which the addition of $\operatorname{HFIP}(0.1,0.5$, and $1.0 \%$ ) to the mobile phases influenced the separation of the three oligonucleotides. The chromatograms are shown in Fig. 2. HFIP clearly affected the retention of the oligonucleotides, but an increase in the HFIP concentration did not improve the absolute resolution. We calculated the resolution of two pairs of oligonucleotides, 18 and 19 mer, and 19 and 20 mer. As shown in Fig. 3, the trends in the $R_{\mathrm{s}}$ values of the two pairs were different. With an increase in the HFIP concentration, the $R_{\mathrm{s}}$ values between 18 and 19 mer increased, while those between 19 and 20 mer decreased. This result indicated that HFIP can change the separation efficiency of oligonucleotides, and that the extent of change depends on the length of the oligonucleotides, and probably, on their sequence.

\section{Effects of column temperature}

In chromatographic separation, the separation selectivity and efficiency are generally influenced by temperature because the separation involves a series of equilibrium reactions where the analytes are either dissolved in the mobile phase or adsorbed 
(a)

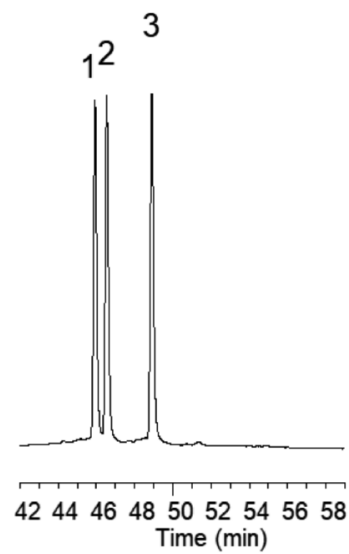

(c)

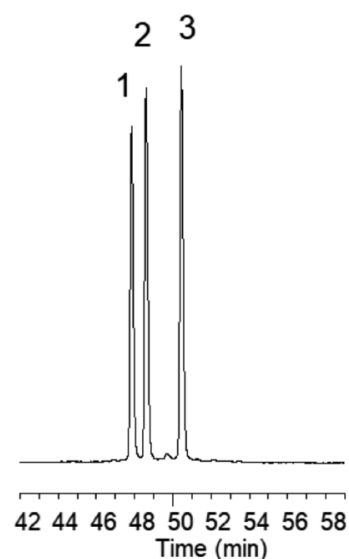

(b)

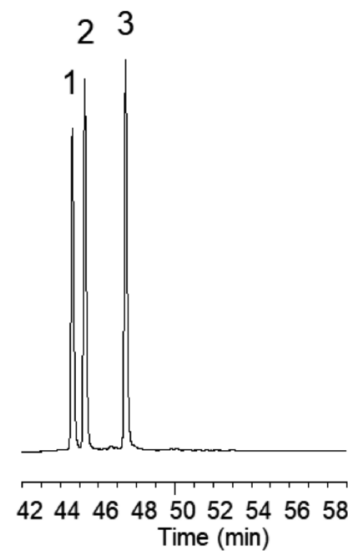

(d)

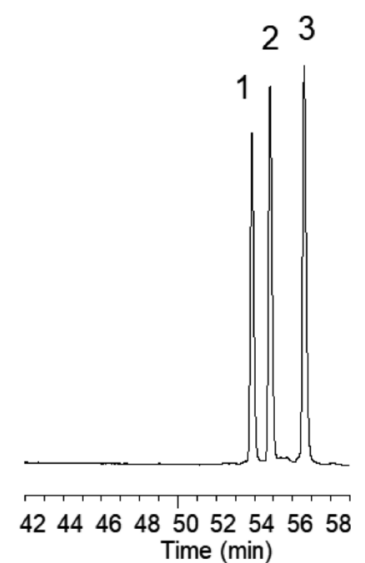

Fig. 2 Effects of the HFIP concentration in mobile phases on the separation of three oligonucleotides: (a) without HFIP, (b) $0.1 \%$ HFIP, (c) $0.5 \%$ HFIP, and (d) $1 \%$ HFIP. Peak identifications are the same as those in Fig. 1.

onto the stationary phase. ${ }^{14}$ The higher is the temperature, the faster is the exchange of analytes between the mobile and stationary phases, and the shorter is the retention time of the analytes on the column. In the case of oligonucleotide separation, high temperatures exceeding $60^{\circ} \mathrm{C}$ are often employed to improve the peak shape and separation. ${ }^{15}$ Although such a high temperature is mandatory for the analysis of doublestranded oligos, such as siRNAs, it is unclear whether the same is also necessary for single-stranded oligonucleotides. Hence, the effect of temperature $\left(40^{\circ} \mathrm{C}\right.$ and $\left.60^{\circ} \mathrm{C}\right)$ on the separation of the three oligonucleotides was evaluated using two different mobile phases (0.1\% and $0.5 \%$ HFIP). As shown in Fig. 4, when the column temperature was increased to $60^{\circ} \mathrm{C}$, shorter retention times, but similar resolutions, were observed with both mobile phases. This suggests that a high temperature is not necessary for separating single-stranded oligonucleotides. Separation at lower temperatures offers several advantages, such as improved column durability and easy scale-up to preparative HPLC.

\section{Effects of stationary phases}

Most studies on oligonucleotide separation have been

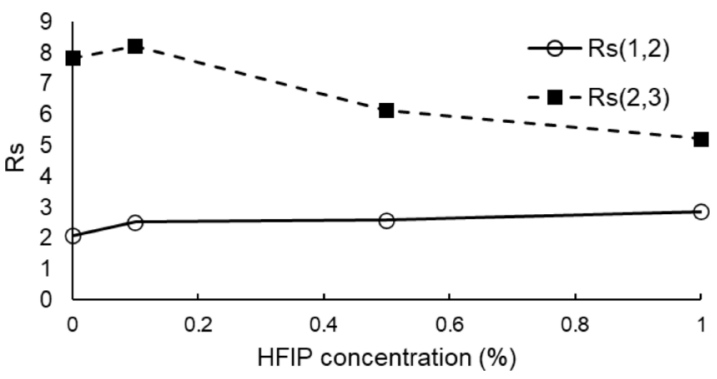

Fig. 3 Relation between the HFIP concentrations and the resolution values (peaks 1 and 2, and peaks 2 and 3 ).

(a)

(b)
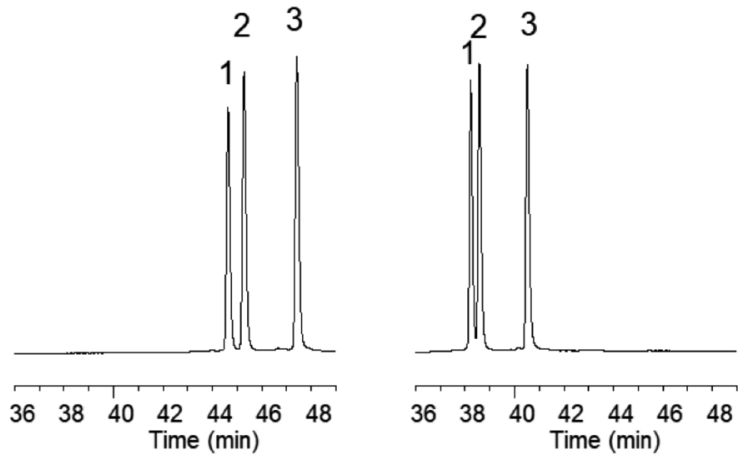

(c)

(d)
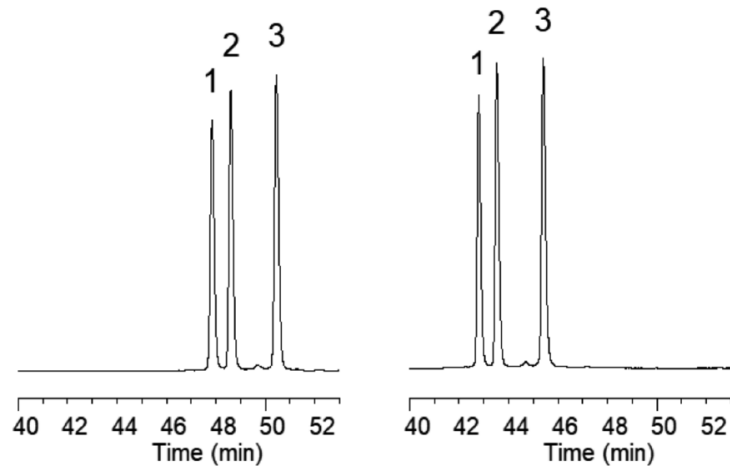

Fig. 4 Effects of the column temperature on the separation of three oligonucleotides: (a) $40^{\circ} \mathrm{C}$ and $0.1 \%$ HFIP, (b) $60^{\circ} \mathrm{C}$ and $0.1 \%$ HFIP, (c) $40^{\circ} \mathrm{C}$ and $0.5 \%$ HFIP, and (d) $60^{\circ} \mathrm{C}$ and $0.5 \%$ HFIP. Peak identifications are the same as those in Fig. 1.

performed using an ODS column. For the analysis of macromolecules, such as proteins, C8 columns are sometimes preferable. Considering that oligonucleotides are not small molecules, a C8 column (InertSustainSwift C8) was investigated instead of an ODS column, with mobile phases containing $0.5 \%$ HFIP at $40^{\circ} \mathrm{C}$. The pore size of the C8 column is $200 \AA$ Figure 5 shows the chromatograms obtained with both the ODS and C8 columns. The retention and resolution of all 
(a)

(b)

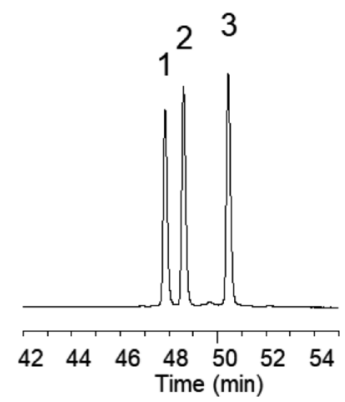

Fig. 5 Effects of stationary phases on the separation of three oligonucleotides. (a) ODS column (IntertSustainSwift C18), and (b) C8 column (InertSustainSwift C8). Peak identifications are the same as those in Fig. 1.

oligonucleotides were similar to those observed for the ODS column. This indicates that the $\mathrm{C} 8$ column provides a separation efficiency similar to that of the ODS column, thus being suitable for oligonucleotide separation.

\section{Conclusions}

With the increasing complexity of oligonucleotide therapeutics, the separation of oligonucleotides is gaining importance. As described in this study, several parameters can influence the separation efficiency of the oligonucleotides. Hence, with further investigation, it should be easier to optimize the separation of oligonucleotide drugs, even if their chemical compositions are different. Since most oligonucleotide-based

drugs are chemically modified, e.g., by phosphorothioate modification, the separation of such oligonucleotides should also be examined.

\section{References}

1. N. Dias and C. A. Stein, Mol. Cancer Ther, 2002, 1, 347.

2. B. D. Adams, C. Parsons, L. Walker, W. C. Zhang, and F. J. Slack, J. Clin. Invest., 2017, 127, 761.

3. C. M. Perry and J. A. Balfour, Drugs, 1999, 57, 375.

4. O. A. Musaimi, D. A. Shaer, F. Albericio, and B. G. de la Torre, Pharmaceuticals, 2021, 14, 145.

5. K. Cook and J. Thayer, Bioanalysis, 2011, 3, 1109.

6. S. M. McCarthy, M. Gilar, and J. Gebler, Anal. Biochem., 2009, 390, 181.

7. M. Gilar, K. J. Fountain, Y. Budman, U. D. Neue, K. R Yardley, P. D. Rainville, R. J. Russell II, and J. C. Gebler, J. Chromatogr. A, 2002, 958, 167.

8. L. W. McLaughlin, Chem. Rev., 1989, 89, 309.

9. A. J. Alpert, J. Chromatogr. A, 1990, 499, 177.

10. L. Gong and J. S. O. McCullagh, J. Chromatogr. A, 2011, 1218,5480

11. A. C. McGinnis, B. Chen, and M. G. Bartlett, J. Chromatogr. $B, 2012,883-884,76$

12. A. P. McKeown, P. N. Shaw, and D. A. Barrett, Chromatographia, 2002, 55, 271.

13. A. Apffel, J. A. Chakel, S. Fischer, K. Lichtenwalter, and W. S. Hancock, Anal. Chem., 1997, 69, 1320.

14. D. Guillarme, S. Heinisch, and J. L. Rocca, J. Chromatogr. A, 2004, 1052, 39.

15. M. Biba, C. J. Welch, J. P. Foley, B. Mao, E. Vazquez, and R. A. Arvary, J. Pharm. Biomed. Anal., 2013, 72, 25. 